\title{
Primary immunodeficiency reveals importance of RIPK1
}

Deficiency of the protein receptor interacting serine/threonine kinase 1 (RIPK1) leads to a compromised immune system in humans that can result in autoimmune clinical features such as arthritis, according to a new study published in Science. RIPK1 is an important regulator of inflammation and cell death. Mice lacking RIPK1 have defects in multiple tissues owing to systemic inflammation and die soon after birth; however, until now the importance of RIPK1 in humans was unknown.

"We studied patients with primary immunodeficiencies and discovered four patients with biallelic loss-of-function mutations in the RIPK1 gene," says Sergey Nejentsev, corresponding author of the study. "This, for the first time, allowed us to characterize effects of complete RIPK1 deficiency in humans."

The four patients had lymphopenia and suffered from recurrent infections (a hallmark of immunodeficiency). Furthermore, they had early-onset inflammatory bowel disease (IBD) and progressive polyarthritis. These autoimmune features resolved in one patient who underwent haematopoietic stem cell transplantation (HSCT).

In vitro, fibroblasts derived from the patients had impaired MAPK signalling, dysregulated cytokine production and were prone to necroptosis (a regulated form of necrosis). Using whole-blood assays, the researchers revealed altered production of multiple cytokines in the absence of RIPK1. In particular, RIPK1-deficient blood cells produced more IL- $1 \beta$ following stimulation with phytohaemagglutinin than blood cells from healthy individuals.

The aberrant production of cytokines, including cytokines implicated in the pathogenesis of arthritis and IBD such as IL- $1 \beta$, might explain the autoimmune

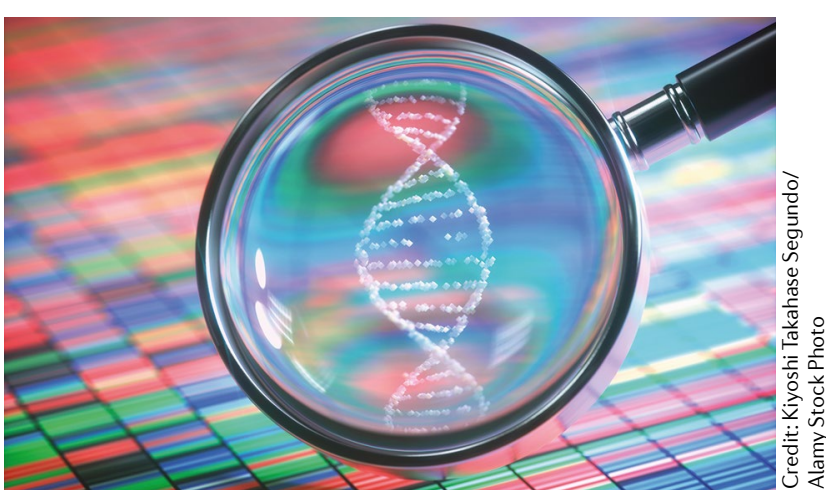

clinical features of RIPK1-deficient patients. Although not tested in this study, the authors postulate that IL-1 inhibitors might be considered for the treatment of such patients.

"An important finding of our study is that effects of RIPK1deficiency in humans are limited

\section{RIPK1 -}

deficient blood cells produced more IL-1 $\beta$... than blood cells from healthy individuals primarily to the immune system," says Nejentsev. "Hence, HSCT can be an effective treatment for such patients if performed early in life."

Jessica McHugh

ORIGINAL ARTICLE Cuchet-Lourenço, D. et al. Biallelic RIPK1 mutations in humans cause severe immunodeficiency, arthritis, and intestinal inflammation. Science. https://doi.org/10.1126/ science.aar2641 (2018)

\section{SYSTEMIC SCLEROSIS}

\section{A fibrosis checkpoint}

"The tyrosine phosphatase SHP2 is a molecular checkpoint for TGF $\beta$ signalling and a potential target for the treatment of fibrosis," says Jörg Distler, corresponding author of a new study published in Nature Communications.

Fibrotic diseases such as systemic sclerosis (SSc) are characterized by persistent and excessive activation of fibroblasts by transforming growth factor- $\beta$ (TGF $\beta$ ), resulting in aberrant deposition of extracellular matrix proteins and consequent scarring and organ dysfunction. However, why pro-fibrotic signalling pathways are unable to turn off is not clear.

"Previous studies from our group and others have demonstrated that transcription factors JAK2 and STAT3 are important downstream mediators of the pro-fibrotic effects of TGF $\beta$ on fibroblasts," says Distler.

In the new work, the researchers identify that SHP2 has a regulatory function in JAK2-STAT3 signalling.
The researchers describe this control as a checkpoint, akin to those that enable cancer cells to avoid the immune system, only here the checkpoint controls persistence of fibrosis.

SHP2 has been shown previously to affect various signalling pathways, and some confusion exists as to whether it activates or deactivates signalling proteins. Importantly, Distler and colleagues now show that TGF $\beta$ has opposing effects on SHP2 expression and activity. In vivo data from patients with SSc, TSK1 mice (which model dermal fibrosis) and a bleomycininduced mouse pulmonary fibrosis model, as well as in vitro stimulation of fibroblasts all show that TGF $\beta$ inhibits expression of SHP2. However, the researchers also show that TGF $\beta$ induces rapid SHP2 phosphatase activity in fibroblasts in vitro. This phosphatase activity is responsible for dephosphorylation of the inhibitory Y570 site in JAK2, resulting in enhanced activation of STAT3 and associated downstream pro-fibrotic signalling.

"The latest results on SHP2 may have direct translational implications as several potent SHP2 inhibitors are available, some of which already showed promising results in first clinical trials in cancer," notes Distler.

Indeed, this new study shows that either fibroblast-specific SHP2 knockout or what might be called checkpoint inhibitors of SHP2 can prevent TGF $\beta$-induced JAK2-STAT3 signalling, fibroblast activation and fibrotic disease in the TSK 1 and bleomycin-induced mouse models. Nicholas J. Bernard activity in fibroblasts 